\title{
Dark pair coherent states of the motion of a trapped ion
}

\author{
S.-C. Gou, J. Steinbach and P.L. Knight \\ Optics Section, Blackett Laboratory, Imperial College, London SW7 2BZ, United Kingdom
}

(March 20, 2018)

\begin{abstract}
We propose a scheme for generating vibrational pair coherent states of the motion of an ion in a two-dimensional trap. In our scheme, the trapped ion is excited bichromatically by three laser beams along different directions in the $X-Y$ plane of the ion trap. We show that if the initial vibrational state is given by a two-mode Fock state, the final steady state, indicated by the extinction of the fluorescence emitted by the ion, is a pure state. The motional state of the ion in the equilibrium realizes that of the highly-correlated pair coherent state.
\end{abstract}

42.50.Vk, 42.50.Dv, 32.80.Pj

Typeset using REVTEX 
A variety of generalized coherent states has been constructed to describe different physical phenomena [1]. Mathematically, the constructions of these sets of generalized coherent states are associated with particular Lie groups. The Glauber coherent states, defined as the right eigenstates of a single-mode boson annihilation operator are associated with the Heisenberg group. Beyond these canonical coherent states, particular generalized coherent states associated with the noncompact $\mathrm{SU}(1,1)$ group have been extensively studied [1 [6]. According to Barut and Girardello [3], these associated coherent states are defined as the eigenstates of the $\mathrm{SU}(1,1)$ lowering operator, whereas in the definition given by Perelomov [4], they are generated by the $\mathrm{SU}(1,1)$ analogue of the displacement operator. These two sets of coherent states are different though they are closely related to the $\mathrm{SU}(1,1)$ group.

Regarding the two-mode boson realization of the SU(1,1) group, a special set of coherent states of the Barut-Girardello type known as pair coherent states (PCS) can be formulated [1]. If $\hat{a}\left(\hat{a}^{\dagger}\right)$ and $\hat{b}\left(\hat{b}^{\dagger}\right)$ denote two independent boson annihilation (creation) operators, then $\hat{a} \hat{b}\left(\hat{a}^{\dagger} \hat{b}^{\dagger}\right)$ stands for the pair annihilation (creation) operator for the two modes. The pair coherent states $|\xi, q\rangle_{\mathrm{PCS}}$ are defined as eigenstates of both the pair annihilation operator $\hat{a} \hat{b}$ and the number difference operator $\hat{Q}=\hat{a}^{\dagger} \hat{a}-\hat{b}^{\dagger} \hat{b}$, i.e.,

$$
\hat{a} \hat{b}|\xi, q\rangle_{\mathrm{PCS}}=\xi|\xi, q\rangle_{\mathrm{PCS}}, \hat{Q}|\xi, q\rangle_{\mathrm{PCS}}=q|\xi, q\rangle_{\mathrm{PCS}},
$$

where $\xi$ is a complex number and $q$ is the "charge" parameter which is a fixed integer. Furthermore, the PCS can be expanded as a superposition of the two-mode Fock states,

$$
|\xi, q\rangle_{\mathrm{PCS}}=\mathcal{N}_{q} \sum_{l=0}^{\infty} \frac{\xi^{l}}{\sqrt{l !(l+q) !}}|l+q, l\rangle_{\mathrm{F}}
$$

where $\mathcal{N}_{q}=\left[|\xi|^{-q} I_{q}(2|\xi|)\right]^{-1 / 2}$ is the normalization constant $\left(I_{q}\right.$ is the modified Bessel function of the first kind of order $q$ ). Pair coherent states were introduced by Horn and Silver [5] to describe the production of pions and applied to other problems in quantum field theory [1]. In quantum optics, PCS are regarded as an important type of correlated two-mode state, which possess prominent nonclassical properties such as sub-Poissonian statistics, correlation in the number fluctuations, squeezing, and violations of Cauchy-Schwartz inequalities [2]. 
The experimental realization of such nonclassical states is of practical importance. Agarwal [2] suggested that the optical PCS can be generated via the competition of 4-wave mixing and two-photon absorption in a nonlinear medium. This is the only scheme proposed to generate PCS known to us. In this Rapid Communication we propose a scheme to generate vibrational PCS of a trapped ion. Recently, due to remarkable advances in laser cooling of a trapped ion [f], it has become possible to realize nonclassical states of the centre-of-mass (CM) motion of a single trapped ion. An ion confined in an electromagnetic trap can be regarded as a particle with quantized CM motion moving in a harmonic potential. Exciting or deexciting the internal atomic states of the trapped ion by a classical laser driving field changes the external states of the ion motion, as atomic stimulated absorption and emission processes are always accompanied by momentum exchange of the laser field with the ion. If both the vibrational amplitude of the ion is much smaller than the laser wavelength, i.e., in the Lamb-Dicke limit [7], and the driving field is tuned to one of the vibrational sidebands of the atomic transition, then this model can be simplified to a form similar to the Jaynes-Cummings model (JCM) [8] in which the quantized radiation field is replaced by the quantized CM motion of the ion. As the coupling between the vibrational modes and the external environment is extremely weak, dissipative effects which are inevitable from cavity damping in the optical regime, can be significantly suppressed for the ion motion. This unique feature thus makes it possible to realize cavity QED experiments without using an optical cavity. Following this approach, nonclassical vibrational states of the trapped ions such as Fock [9], squeezed [10] and Schrödinger cat states [11,12] have been proposed and observed [13].

Consider the quantized motion of a two-level ion of mass $M$ which is trapped in a twodimensional (2-D) isotropic harmonic potential characterized by the trap frequency $\nu$. The creation (annihilation) of vibrational quanta in the $X$ and $Y$ directions is described by the operators $\hat{a}\left(\hat{a}^{\dagger}\right)$ and $\hat{b}\left(\hat{b}^{\dagger}\right)$ respectively. The position operators are given by $\hat{x}=\lambda\left(\hat{a}+\hat{a}^{\dagger}\right)$ and $\hat{y}=\lambda\left(\hat{b}+\hat{b}^{\dagger}\right)$, where $\lambda=\sqrt{\hbar / 2 \nu M}$. In our scheme, which we sketch in Fig. 1, the ion is driven bichromatically by three laser beams in the $X-Y$ plane. The first two lasers are both tuned 
to the second lower vibrational sideband and applied to the ion along directions with an angle $\pi / 4$ and $3 \pi / 4$ relative to the $X$-axis, respectively. The third laser which drives the ion along the $X$-axis is resonant with the atomic transition frequency. In the rotating-wave approximation, the Hamiltonian describing the coherent evolution is

$$
\hat{H}=\hbar \nu\left(\hat{a}^{\dagger} \hat{a}+\hat{b}^{\dagger} \hat{b}\right)+\frac{\hbar \omega_{0}}{2} \hat{\sigma}_{z}-\left[\mathcal{D} E^{(-)}(\hat{x}, \hat{y}, t) \hat{\sigma}_{-}+\text {h.c. }\right]
$$

where the first two terms describe the free evolution of the external and internal degrees of freedom of the ion and the last indicates the atom-field interaction. The operators $\hat{\sigma}_{+}$and $\hat{\sigma}_{-}$are raising and lowering operators for the two-level ion obeying commutation relations $\left[\hat{\sigma}_{+}, \hat{\sigma}_{-}\right]=\hat{\sigma}_{z}$ and $\left[\hat{\sigma}_{z}, \hat{\sigma}_{ \pm}\right]= \pm 2 \hat{\sigma}_{ \pm}$. The transition in the two-level ion is characterized by the dipole matrix element $\mathcal{D}$ and the transition frequency $\omega_{0}$. The negative frequency part of the classical electric driving field is given by

$$
\begin{aligned}
E^{(-)}(\hat{x}, \hat{y}, t)= & E_{1} e^{i\left[\left(\omega_{0}-2 \nu\right) t-k_{2} \hat{x}^{\prime}+\phi_{1}\right]}+E_{2} e^{i\left[\left(\omega_{0}-2 \nu\right) t-k_{2} \hat{y}^{\prime}+\phi_{2}\right]} \\
& +E_{0} e^{i\left(\omega_{0} t-k_{0} \hat{x}+\phi_{0}\right)}
\end{aligned}
$$

where $2 E_{j}$ and $\phi_{j}$ indicate the amplitudes and phases of the driving lasers respectively. We have introduced new position operators $\hat{x}^{\prime}$ and $\hat{y}^{\prime}$ which are related to $\hat{x}$ and $\hat{y}$ by a $\pi / 4$ rotation in the $X-Y$ plane,

$$
\left(\begin{array}{l}
\hat{x}^{\prime} \\
\hat{y}^{\prime}
\end{array}\right)=\frac{1}{\sqrt{2}}\left(\begin{array}{cc}
1 & 1 \\
-1 & 1
\end{array}\right)\left(\begin{array}{l}
\hat{x} \\
\hat{y}
\end{array}\right),
$$

so that for the creation (annihilation) operators $\hat{A}\left(\hat{A}^{\dagger}\right)$ and $\hat{B}\left(\hat{B}^{\dagger}\right)$, defined in the $X^{\prime}$ and $Y^{\prime}$ directions respectively, we obtain the following transformation

$$
\left(\begin{array}{l}
\hat{A} \\
\hat{B}
\end{array}\right)=\frac{1}{\sqrt{2}}\left(\begin{array}{cc}
1 & 1 \\
-1 & 1
\end{array}\right)\left(\begin{array}{l}
\hat{a} \\
\hat{b}
\end{array}\right) .
$$

According to Vogel and de Matos Filho [11,[1], if the ion is in the resolved sideband limit and the driving laser is resonant with one of the vibrational sidebands, then the ion-laser 
interaction can be described as a nonlinear JCM. Following Refs. [11, 14, the Hamiltonian of Eq.(3) can be written in the interaction picture as

$$
\begin{aligned}
\hat{H}_{\mathrm{I}}= & \hbar e^{-\eta^{2} / 2}\left[\sum_{j=0}^{\infty} \frac{(i \eta)^{2 j+2}}{j !(j+2) !}\left[\Omega_{1} e^{i \phi_{1}} \hat{A}^{j}\left(\hat{A}^{\dagger}\right)^{j+2}+\Omega_{2} e^{i \phi_{2}} \hat{B}^{j}\left(\hat{B}^{\dagger}\right)^{j+2}\right]\right. \\
& \left.+\Omega_{0} e^{i \phi_{0}} \sum_{j=0}^{\infty} \frac{(i \eta)^{2 j}}{j ! j !} \hat{a}^{j}\left(\hat{a}^{\dagger}\right)^{j}\right] \hat{\sigma}_{-}+\text {h.c. }
\end{aligned}
$$

where $\Omega_{j}=-\mathcal{D} E_{j} / \hbar$ are the Rabi frequencies, and the Lamb-Dicke parameter $\eta=k \lambda$ has been defined assuming $k_{2} \simeq k_{0}=k$.

As the damping of vibrational quanta can be significantly suppressed in an ion trap, the dominant decay process is the spontaneous emission from the two-level ion, and the time evolution of the system in the interaction picture can be described by a density operator $\hat{\rho}$ obeying the master equation 9, 11

$$
\frac{d \hat{\rho}}{d t}=-\frac{i}{\hbar}\left[\hat{H}_{\mathrm{I}}, \hat{\rho}\right]+\frac{\Gamma}{2}\left(2 \hat{\sigma}_{-} \hat{\varrho} \hat{\sigma}_{+}-\hat{\sigma}_{+} \hat{\sigma}_{-} \hat{\rho}-\hat{\sigma}_{-} \hat{\sigma}_{+} \hat{\rho}\right)
$$

where $\Gamma$ is the spontaneous decay rate of the excited state of the ion, and the modified density operator

$$
\hat{\varrho}=\frac{1}{4} \int_{-1}^{1} \int_{-1}^{1} d u d v W(u, v) e^{i k(u \hat{x}+v \hat{y})} \hat{\rho} e^{-i k(u \hat{x}+v \hat{y})}
$$

accounts for the momentum transfer in the $X-Y$ plane due to spontaneous emission, where $W(u, v)$ describes the angular distribution of the spontaneous emission. In the Lamb-Dicke regime, $\eta \ll 1$, the master equation, Eq.(8), can be well approximated by the first order expansion in $\eta$. In this case, only the leading terms, i.e., $j=0$ in $\hat{H}_{\mathrm{I}}$, are considered, and $\hat{\varrho}$ is replaced by $\hat{\rho}$. Setting $\Omega_{1}=\Omega_{2}=\Omega$, and $\phi_{1}=0, \phi_{2}=\pi$, the master equation Eq.(8) simplifies to

$$
\frac{d \hat{\rho}}{d t}=-\frac{i}{\hbar}\left[\hat{H}_{\mathrm{I}}^{\prime}, \hat{\rho}\right]+\frac{\Gamma}{2}\left(2 \hat{\sigma}_{-} \hat{\rho} \hat{\sigma}_{+}-\hat{\sigma}_{+} \hat{\sigma}_{-} \hat{\rho}-\hat{\sigma}_{-} \hat{\sigma}_{+} \hat{\rho}\right)
$$

with the effective Hamiltonian $\hat{H}_{\mathrm{I}}^{\prime}$ given by

$$
\hat{H}_{\mathrm{I}}^{\prime}=\alpha[\hat{a} \hat{b}-\xi] \hat{\sigma}_{+}+\text {h.c. }
$$


where $\alpha=\hbar \Omega \eta^{2} \exp \left(-\eta^{2} / 2\right)$, and $\xi=\Omega_{0} \Omega^{-1} \eta^{-2} \exp \left(-i \phi_{0}\right)$. Disregarding the constant driving term $\xi$, the effective Hamiltonian of Eq.(11) is identical to the non-degenerate twomode JCM [15].

For a master equation of the form of Eq.(10), the steady-state solution $\hat{\rho}_{\mathrm{s}}$ is a pure state [9]11]

$$
\hat{\rho}_{\mathrm{s}}=|g\rangle|\psi\rangle\langle\psi|\langle g|,
$$

where $|g\rangle$ is the atomic ground state and $|\psi\rangle$ defines the vibrational state of the ion. When the system reaches the steady state $d \hat{\rho}_{\mathrm{s}} / d t=0$. Assuming a steady state of the form above gives $\left[\hat{H}_{\mathrm{I}}^{\prime}, \hat{\rho}_{\mathrm{s}}\right]=0$, as the dissipative term on the right hand side of Eq.(10) vanishes in this case. Consequently, a sufficient condition for us to generate the vibrational steady state $|\psi\rangle$ is to ensure $\hat{\rho}_{\mathrm{s}}$ satisfies the restriction $\left[\hat{H}_{\mathrm{I}}^{\prime}, \hat{\rho}_{\mathrm{s}}\right]=0$, or equivalently $\hat{a} \hat{b}|\psi\rangle=\xi|\psi\rangle$. Evidently, this is identical to our first definition of a PCS given above in Eq.(1). In order to generate a fully characterized PCS, however, we need another constraint to ensure that the final vibrational state remains an eigenstate of the number difference operator $\hat{Q}$ [Eq.(1)]. This can be achieved by properly choosing the initial vibrational state. In view of the fact that $[\hat{a} \hat{b}, \hat{Q}]=0$, it follows that the number difference is a constant of motion in processes involving simultaneous pair annihilation or creation. Thus for an initial vibrational state $\left|\psi_{0}\right\rangle$, if the conserved "charge" condition is initially satisfied, i.e., $\hat{Q}\left|\psi_{0}\right\rangle=q\left|\psi_{0}\right\rangle$, then this condition holds for the whole time evolution of the system described by Eq.(10). Regarding the feasibility of an experimental realization, it seems that the two-mode Fock state $\left|\psi_{0}\right\rangle=$ $|m+q, m\rangle_{\mathrm{F}}$ is best suited to fulfil the conserved "charge" condition. Without loss of generality we may set the initial vibrational state as $\left|\psi_{0}\right\rangle=|q, 0\rangle_{\mathrm{F}}(q \geq 0)$ which, according to recent work, can be prepared with very high efficiency [13].

In order to identify the validity of our analytic argument and to gain insight into the transient behaviour we have solved the master equation, Eq.(10), numerically employing a Monte-Carlo state-vector method [16]. Our numerical analysis was performed using a highorder unravelling technique [17] in a finite (truncated) Fock state basis with a cut-off chosen 
such that an increase of this cut-off does not alter the result of our integration.

We find that the system evolves from an initial product of a two-mode Fock state and a superposition of the internal states of the ion into the pure steady state [Eq.(12)] as expected. Fig.2 depicts the excitation number distribution in the two vibrational modes at different times, showing the system as it evolves from the initial pure state $\left|\Psi_{0}\right\rangle=|e\rangle \otimes|7,6\rangle_{\mathrm{F}}$ into its steady state. The steady-state excitation number distribution [Fig.2(d)] is indistinguishable from that of a PCS with $\xi=2$ and $q=1$. This is the expected result since the particular choice of the initial state determines the conserved "charge" $q=\left\langle\Psi_{0}|\hat{Q}| \Psi_{0}\right\rangle=1$, and from Eqs.(1) and (11) the second number defining the PCS is determined by the driving term $\xi$ in the effective Hamiltonian, which in the example shown is $\xi=2$. We verify that the steady state is pure by calculating the trace of the square of the density operator $\operatorname{Tr}\left(\hat{\rho}_{\mathrm{s}}^{2}\right)$ from the steady state obtained in our numerical integration. We find $\operatorname{Tr}\left(\hat{\rho}_{\mathrm{s}}^{2}\right)=0.9997$. Fig. 3 shows the transient behaviour of the internal state of the ion. We depict the time evolution of the inversion, $\left\langle\hat{\sigma}_{z}\right\rangle$, [Fig. $\left.\cdot 3(\mathrm{a})\right]$ and the imaginary part of the polarization, $i\left\langle\hat{\sigma}_{-}-\hat{\sigma}_{+}\right\rangle$, [Fig. $\left.3(\mathrm{~b})\right]$. We note the timescale for the system to reach the steady state is much longer than it would take the same ion to reach its steady state in a resonance fluorescence experiment.

One may examine the existence of the PCS by observing the collapses and the revivals of the atomic inversion. This is illustrated by switching off the carrier field suddenly once the system has reached the steady state. In this case, the system is equivalent to the nondegenerate two-mode JCM interacting with a PCS when the ion is initially in its ground state. The time evolution of such a system has been investigated by several authors 18]. In particular, when $q=0$ the Rabi oscillation of the atom exhibits exactly periodic behaviour. However, as the atomic decay is essential to the present treatment and cannot be ignored, the collapses and revivals of the Rabi oscillation of the trapped ion are substantially different from the previous results. One possible way to suppress the complications caused by the atomic decay is to increase the intensities of the driving lasers so that the ratio $\Gamma / \alpha$ is lowered. Thus, in the short time regime the influence of spontaneous emission can be eliminated.

In conclusion, we have proposed a scheme for the realization of pair coherent states of 
the CM motion of a trapped ion. In our scheme, three laser beams, one of which is tuned to the carrier frequency and the other two to the second lower vibrational sideband, are used to drive the ion trapped in a 2-D isotropic harmonic potential well. In appropriate limits, the system will relax to a steady state due to the spontaneous emission from the ion. If the vibrational state of motion of the ion is initially prepared in a Fock state, then the steady state of the system is a pure state given by a product of the atomic ground state with a PCS of the vibrational motion. In this case, the two parameters, $\xi$ and $q$, that characterize the PCS are determined by the intensities and phases of the driving lasers and by the number difference between the two vibrational modes determined by the initial Fock state respectively.

This work was supported in part by the UK Engineering and Physical Sciences Research Council and the European Union. S.-C. Gou acknowledges support from the Ministry of Education, Taiwan, Republic of China. J. Steinbach is supported by the German Academic Exchange Service (DAAD-Doktorandenstipendium aus Mitteln des zweiten Hochschulsonderprogramms). 


\section{REFERENCES}

[1] J.R. Klauder and B. Skagerstam, Coherent States - Applications in Physics and Mathematical Physics (World Scientific, Singapore, 1985).

[2] G.S. Agarwal, J. Opt. Soc. Am. B 5, 1940 (1988); G.S. Agarwal, Phys. Rev. Lett. 57, 827 (1986).

[3] A.O. Barut and L. Girardello, Commun. Math. Phys. 21, 41 (1971).

[4] A.M. Perelomov, Generalized Coherent States and their Applications (Springer-Verlag, Berlin, 1985).

[5] D. Horn and R. Silver, Phys. Rev. D 2, 2082 (1970).

[6] V. Bužek, J. Mod. Opt. 37, 303 (1990); C.C. Gerry, J. Opt. Soc. Am. B 8, 685 (1991);

L. Gilles and P.L. Knight, J. Mod. Opt. 39, 1411 (1992).

[7] F. Diedrich, J.C. Bergquist, W.M. Itano, and D.J. Wineland, Phys. Rev. Lett. 62, 403 (1989); C. Monroe, D.M. Meekhof, B.E. King, S.R. Jefferts, W.M. Itano, and D.J. Wineland, Phys. Rev. Lett. 75, 4011 (1995).

[8] C.A. Blockley, D.F. Walls, and H. Risken, Europhys. Lett. 17, 509 (1992).

[9] J.I. Cirac, R. Blatt, A.S. Parkins, and P. Zoller, Phys. Rev. Lett. 70, 762 (1993); J.I. Cirac, R. Blatt, and P. Zoller, Phys. Rev. A 49, R3174 (1994).

[10] J.I. Cirac, A.S. Parkins, R. Blatt, and P. Zoller, Phys. Rev. Lett. 70, 556 (1993).

[11] R.L. de Matos Filho and W. Vogel, Phys. Rev. Lett. 76, 608 (1996).

[12] J.F. Poyatos, J.I. Cirac, R. Blatt, and P. Zoller, submitted (1995); C. Monroe, D.M. Meekhof, B.E. King, and D.J. Wineland, submitted to Science (1996).

[13] D.M. Meekhof, C. Monroe, B.E. King, W.M. Itano, and D.J. Wineland, Phys. Rev. Lett. 76, 1796 (1996). 
[14] W. Vogel and R.L. de Matos Filho, Phys. Rev. A 52, 4214 (1995).

[15] S.-C. Gou, Phys. Rev. A 40, 5116 (1989).

[16] J. Dalibard, Y. Castin and K. Mølmer, Phys. Rev. Lett. 68, 580 (1992); H.J. Carmichael, An Open System Approach to Quantum Optics, Lecture Notes in Physics (SpringerVerlag, Berlin, 1993); R. Dum, P. Zoller and H. Ritsch, Phys. Rev. A 45, 4879 (1992); G.C. Hegerfeldt and T.S. Wilser, Proceedings of the II. International Wigner Symposium, Goslar 1991; H.D. Doebner, W. Scherer and F. Schroeck Eds. (World Scientific, Singapore, 1992).

[17] J. Steinbach, B.M. Garraway and P.L. Knight, Phys. Rev. A 51, 3302 (1995).

[18] S.-C. Gou, J. Mod. Opt. 37, 1469 (1990); A. Joshi and R.R. Puri, Phys. Rev. A 42, 4336 (1990). 


\section{FIGURES}

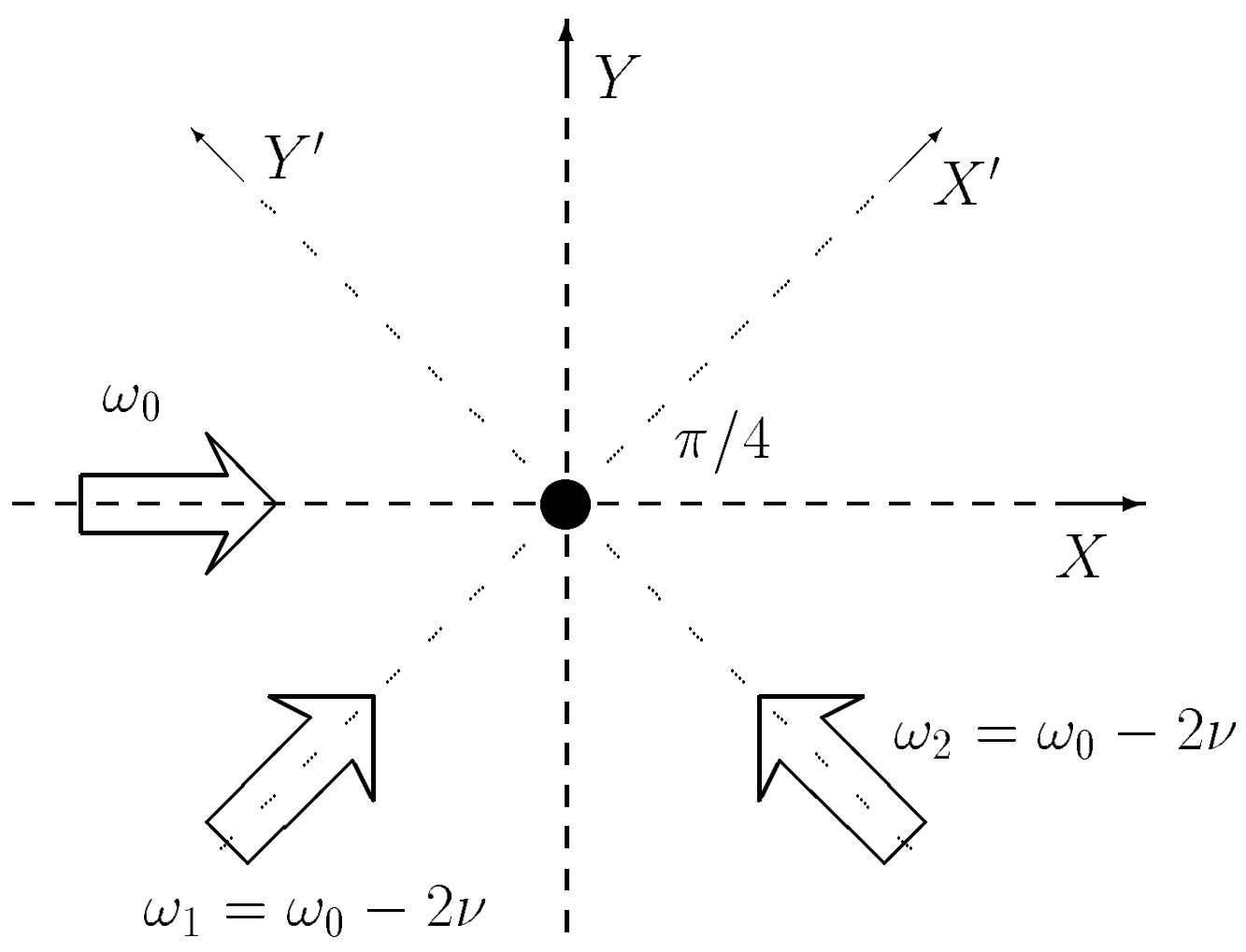

FIG. 1.

Configuration to generate vibrational PCS. The carrier field drives the ion on resonance along the $X$-axis. The other two lasers are both resonant with the second lower vibrational sideband. They drive the ion along the $X^{\prime}$ and $Y^{\prime}$-axis having phases $\phi_{1}=0$ and $\phi_{2}=\pi$ respectively. 
(a)

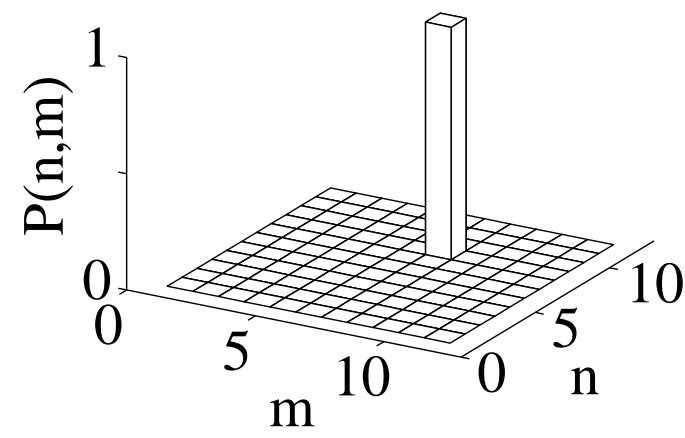

(c)

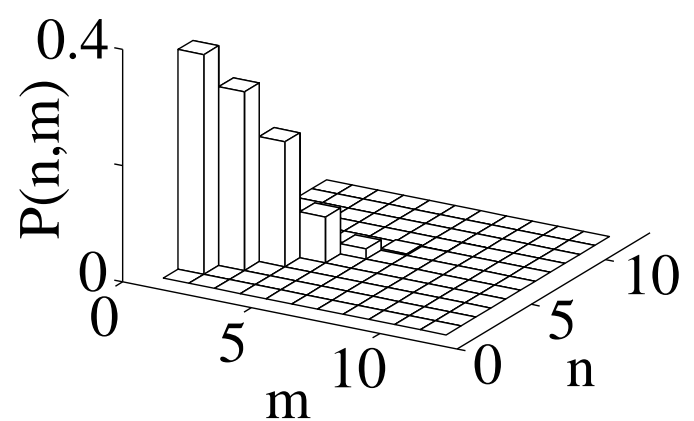

(b)

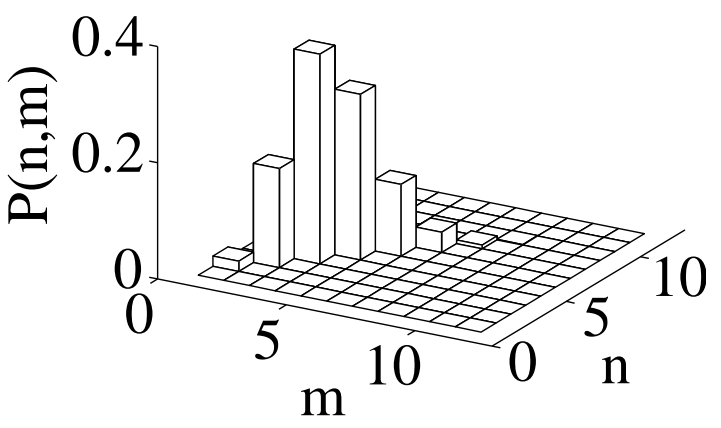

(d)

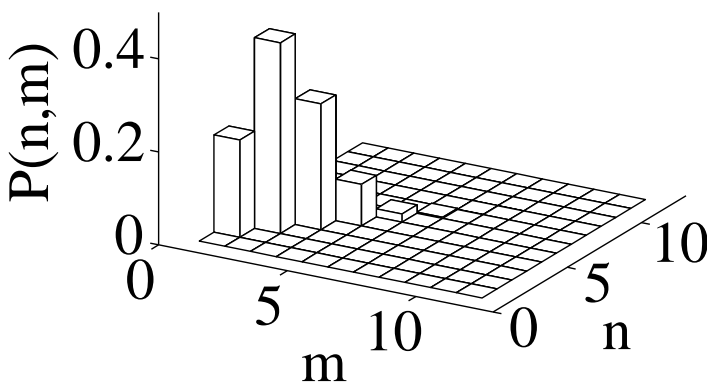

FIG. 2.

We depict the excitation number distribution $\mathrm{P}(\mathrm{n}, \mathrm{m})$ in the two vibrational modes at different times. The system evolves from (a), the initial pure state $\left|\Psi_{0}\right\rangle=|e\rangle \otimes|7,6\rangle_{\mathrm{F}}$ $(\Gamma t=0.0)$, through $(\mathrm{b})$ and $(\mathrm{c})$, two intermediate states $(\Gamma t=125.0)$ and $(\Gamma t=500.0)$, into (d), its steady state which is the pure product of the internal ground state of the ion and a vibrational PCS: $\left|\Psi_{\mathrm{s}}\right\rangle=|g\rangle \otimes|2,1\rangle_{\mathrm{PCS}}(\Gamma t=2000.0)$. The data shown has been obtained from a Monte-Carlo simulation which included 1000 trajectories and was performed in a truncated Fock state basis $\left(|0,0\rangle_{\mathrm{F}} \ldots|20,20\rangle_{\mathrm{F}}\right)$. Parameters: $\alpha=0.2, \xi=2.0, \Gamma=10.0$. 


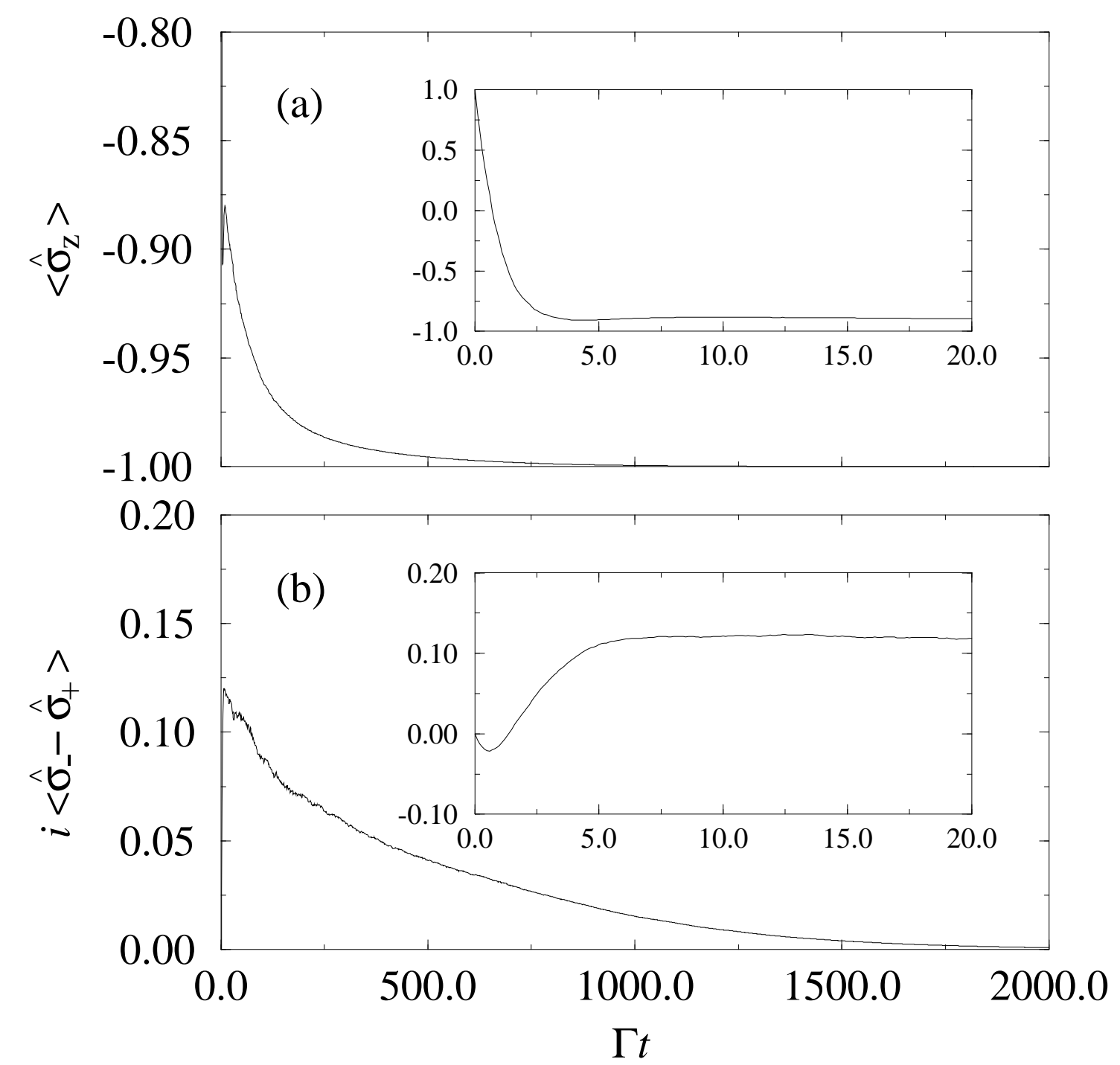

FIG. 3.

Time evolution of the internal state of the two-level ion as the system evolves into its steady state. (a) shows the inversion, $\left\langle\hat{\sigma}_{z}\right\rangle$, and (b) the imaginary part of the polarization, $i\left\langle\hat{\sigma}_{-}-\hat{\sigma}_{+}\right\rangle$. The real part of the polarization, $\left\langle\hat{\sigma}_{-}+\hat{\sigma}_{+}\right\rangle$, remains zero at all times. The two insets show the evolution in the time interval $\Gamma t=0.0$ to $\Gamma t=20.0$. Parameters and data used are the same as in Fig.2. 